\title{
Terahertz Wide-Angle Imaging and Analysis on Plane-wave Criteria Based on Inverse Synthetic Aperture Techniques
}

\author{
Jing Kun Gao ${ }^{1} \cdot$ Yu Liang Qin ${ }^{1} \cdot$ Bin Deng $^{1}$ •

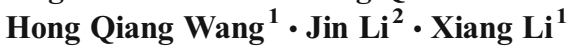

Received: 22 July 2015 / Accepted: 5 January 2016 /

Published online: 22 January 2016

(C) The Author(s) 2016. This article is published with open access at Springerlink.com

\begin{abstract}
This paper presents two parts of work around terahertz imaging applications. The first part aims at solving the problems occurred with the increasing of the rotation angle. To compensate for the nonlinearity of terahertz radar systems, a calibration signal acquired from a bright target is always used. Generally, this compensation inserts an extra linear phase term in the intermediate frequency (IF) echo signal which is not expected in large-rotation angle imaging applications. We carried out a detailed theoretical analysis on this problem, and a minimum entropy criterion was employed to estimate and compensate for the linear-phase errors. In the second part, the effects of spherical wave on terahertz inverse synthetic aperture imaging are analyzed. Analytic criteria of plane-wave approximation were derived in the cases of different rotation angles. Experimental results of corner reflectors and an aircraft model based on a $330-\mathrm{GHz}$ linear frequency-modulated continuous wave (LFMCW) radar system validated the necessity and effectiveness of the proposed compensation. By comparing the experimental images obtained under plane-wave assumption and spherical-wave correction, it also showed to be highly consistent with the analytic criteria we derived.
\end{abstract}

Keywords Terahertz imaging $\cdot$ Large rotation angle $\cdot$ Plane-wave criteria $\cdot$ Inverse synthetic aperture radar (ISAR)

Jing Kun Gao

oscar92923@163.com

1 Institute of Space Electronic Technology, National University of Defense Technology, Changsha, China

2 School of Electronic Engineering, University of Electronic Science and Technology of China, Chengdu, China 


\section{Introduction}

Terahertz $(\mathrm{THz})$ wave has many distinctive characteristics as its wavelength lies between microwave and infrared wave. Terahertz imaging techniques have drew much attention in the field of terahertz applications due to several exciting advantages of terahertz wave. The lower photon energy property makes it safer to take safety inspection on the human body and harmless detection of biological samples. The penetration ability towards dielectric and liquid nonpolar materials makes it able to realize perspective imaging. The shorter wavelength and higher frequency provide the ability to realize wider absolute bandwidth and higher spatial resolution. All these characteristics lead to promising prospects in nondestructive detection [1], medical imaging [2,3], and standoff personnel screening [4-6].

Recently, great progress has been made on THz radar systems. One of the typical institutions is the Jet Propulsion Laboratory (JPL). In 2006, they successfully developed the first high-resolution terahertz imaging radar with a 2-cm range resolution [7, 8]. In 2011, subcentimeter resolution in a 4-25-m distance and appreciable imaging speed were already available $[4,6,9,10]$. Another representative is the German Fraunhofer Institute for High Frequency Physics and Radar Techniques (FHR). In 2007, they successfully developed a 220$\mathrm{GHz}$ terahertz imaging radar system COBRA-220 [11] which realized a 1.8-cm resolution. In 2013, they continuously developed a 300-GHz system which obtained a 3.7-mm resolution [12]. Beside these two forerunners, more and more organizations started to develop their own systems and many remarkable achievements had been made [13-23].

In most of these works, a linear frequency-modulated continuous wave (LFMCW) mode was introduced. It is easier for LFMCW radars to realize a wider bandwidth as well as to lower the emission power. The stretch processing can reduce the intermediate frequency (IF) bandwidth while maintaining the range resolution, which helps to simplify the system design and reduce the cost. LFMCW signals are also of stable coherence which is extremely important when inverse synthetic aperture radar (ISAR) techniques are utilized to generate high-resolution $2 \mathrm{D}$ or $3 \mathrm{D}$ images.

However, to obtain higher resolution through ISAR techniques, some problems still need to be considered carefully. Firstly, problems occurred with the increasing of the rotation angle can damage the focusing of images. As it is hard to realize prefect linearity within the total broad bandwidth of the LFM signal in the terahertz regime, a calibration signal acquired from a bright target is always used to compensate for the nonlinearity of the frequency modulation rate $[4,23,24]$. Generally, a deviation distance between the reference bright target and the equivalent center of turntable always exists in real cases. This deviation distance leads to an extra linear phase term in the compensated IF signal. In the cases of large rotation angle, this extra term results in serious defocus of the image. Secondly, as we know, terahertz radar has shorter wavelength compared to traditional microwave radar. In addition, the interval between radar and target is usually several meters to tens meters in terahertz imaging applications and the atmospheric attenuation also confines the propagation distance. To date, no detailed analysis on the effects that these problems bring to ISAR imaging at terahertz frequencies has been carried out yet.

In this paper, two parts of work were presented around imaging applications based on a 330-GHz LFMCW radar system [24]. Firstly, the problems occurred with the increasing of the rotation angle were discussed. In order to improve the resolution and the image Signal to Noise Ratio (SNR) by utilizing the large rotation angle, it is necessary to compensate for this extra phase term and realize the coherent addition of the data acquired from different apertures. 
Detailed theoretical derivation is shown in this paper. Careful analysis was taken to reveal the impact to the quality of images that caused by deviation distance and large rotation angle. A minimum entropy criterion was used to estimate and correct this deviation distance. Experimental results validated the necessity and effectiveness of this compensation. Secondly, a detailed theoretical analysis on the spherical-wave effects was carried out. The criteria of plane-wave approximation were derived in different cases, and experimental results showed great consistency with the theoretical analysis.

The remainder of this paper is organized as follows: Section 2 describes the imaging problem occurred with the increasing of rotation angle in THz LFMCW radar systems and the solution of this problem. Section 3 analyzes the effect of spherical wave and provides the criteria of plane-wave approximation. Section 4 gives the experimental results. Finally, we conclude this paper in Section 5.

\section{THz LFMCW Large Rotation Angle Imaging}

\subsection{Theoretical Derivation}

Suppose a LFM signal is transmitted and a stretch processing is employed. The ideal received IF signal scattered from a point target should be a perfect sinusoid, and its frequency is determined by the distance between the radar and the target. But there are always phase noises in the real echo IF signals which insert distortion into the sinusoid. Figure 1 shows the basic geometric relationships. Suppose an ideal point target is $R_{r}$ away from the radar and then the echo IF signal with phase noises will be [25]

$s_{r}\left(\tau, t_{m}\right)=A \cdot \operatorname{rect}\left(\frac{\tau-2 R_{r} / c}{T_{p}}\right) \exp \left(-j 4 \pi f_{c} R_{\Delta}^{r} / c-j 4 \pi \gamma\left(\tau-2 R_{r} / c\right) R_{\Delta}^{r} / c-j 4 \pi \gamma R_{\Delta}^{r}{ }^{2} / c^{2}+j \varphi\left(\tau-2 R_{\mathrm{ref}} / c, R_{\Delta}^{r}\right)\right)$

where $R_{\Delta}^{r}=R_{r}-R_{\text {ref, }}, R_{\text {ref }}$ is the reference distance selected by the radar system (not shown in Fig. 1), $\gamma$ is the frequency modulation rate, and $\varphi\left(\tau-2 R_{\text {ref }} / c, R_{\Delta}^{r}\right)$ represents the phase noises which is a function of both fast time and the distance between the radar and the target. $\varphi\left(\tau-2 R_{\text {ref }} / c, R_{\Delta}^{r}\right)$ can be approximated as a constant function of $R_{\Delta}^{r}$ when the size of the target

Fig. 1 Basic geometry

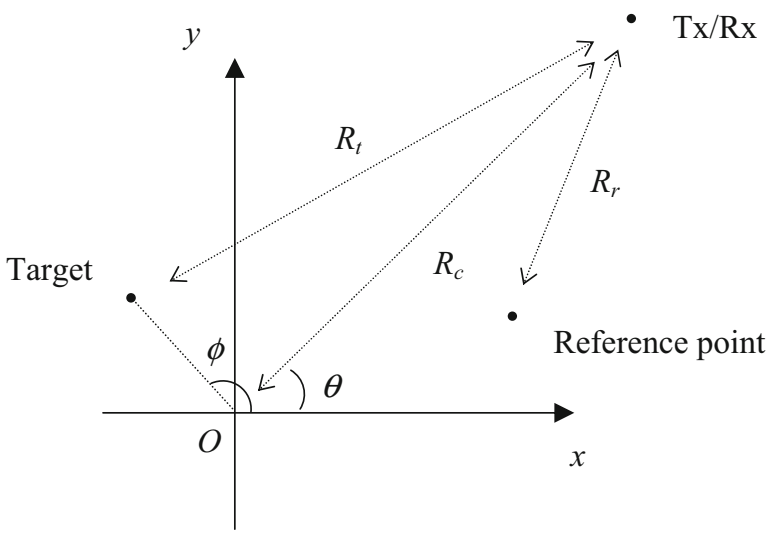


is much smaller than the spatial length of the transmitted signal which means that $\varphi\left(\tau-2 R_{\text {ref }} f, R_{\Delta}^{r}\right)$ varies slowly against $R_{\Delta}^{r}$ as the change of $R_{\Delta}^{r}$ is small. For the convenience of derivation, we redefine the fast time as $\tau={ }^{\Delta} \tau-2 R_{\text {ref }} / c$

$s_{r}\left(\tau, t_{m}\right)=A_{r} \cdot \operatorname{rect}\left(\frac{\tau-2 R_{\Delta}^{r} / c}{T_{p}}\right) \exp \left(-j 4 \pi f_{c} R_{\Delta}^{r} / c-j 4 \pi \gamma\left(\tau-2 R_{\Delta}^{r} / c\right) R_{\Delta}^{r} / c-j 4 \pi \gamma R_{\Delta}^{r^{2}} / c^{2}+j \varphi\left(\tau, R_{\Delta}^{r}\right)\right)$

Similarly, the echo IF signal of a target located $R_{t}$ away from radar is

$$
s_{t}\left(\tau, t_{m}\right)=A_{t} \cdot \operatorname{rect}\left(\frac{\tau-2 R_{\Delta}^{t} / c}{T_{p}}\right) \exp \left(-j 4 \pi f_{c} R_{\Delta}^{t} / c-j 4 \pi \gamma\left(\tau-2 R_{\Delta}^{t} / c\right) R_{\Delta}^{t} / c-j 4 \pi \gamma R_{\Delta}^{t}{ }^{2} / c^{2}+j \varphi\left(\tau, R_{\Delta}^{t}\right)\right)
$$

where $R_{\Delta}^{t}=R_{t}-R_{\text {ref }}$; use $s_{r}\left(\tau, t_{m}\right)$ as the calibration signal to compensate the echo signal of target

$$
\begin{aligned}
s_{t r}\left(\tau, t_{m}\right) & =s_{t}\left(\tau, t_{m}\right) / s_{r}\left(\tau, t_{m}\right) \\
& =A_{t} / A_{r} \cdot \operatorname{rect}\left(\frac{\tau-2 R_{\Delta} / c}{T_{p}}\right) \operatorname{rect}\left(\frac{\tau}{T_{p}}\right) \cdot \exp \left(-j 4 \pi f_{c} R_{\Delta} / c-j 4 \pi \gamma\left(\tau-2 R_{\Delta} / c\right) R_{\Delta} / c-j 4 \pi \gamma R_{\Delta}^{2} / c^{2}\right)^{(4)}
\end{aligned}
$$

where $R_{\Delta}=R_{t}-R_{r}$. After the procedure of ramp phase and residual video-phase (RVP) correction, the signal becomes

$$
\begin{aligned}
s_{t c}\left(\tau, t_{m}\right) & =\operatorname{IFT}\left(\operatorname{FT}\left(s_{t r}\left(\tau, t_{m}\right)\right) \cdot \exp \left(-j \pi f^{2} / \gamma\right)\right) \\
& =A_{t} / A_{r} \cdot \operatorname{rect}\left(\frac{\tau}{T_{p}}\right) \cdot \exp \left(-j 4 \pi\left(f_{c}+\gamma \tau\right) R_{\Delta} / c\right)
\end{aligned}
$$

Suppose the range alignment and phase correction have already been accomplished for moving targets and the echo signal model is equivalent to a standard turntable model. Under the plane-wave approximation, $R_{t}$ will be

$$
R_{t}=R_{c}-(x \cos \theta+y \sin \theta)
$$

Meanwhile, utilizing the relationship $f=f_{c}+\gamma \tau$ and $k=\frac{2 \pi f}{c}$ where $f$ represents the real time frequency for the LFM signal and $k$ stands for the wavenumber, Eq. (5) can be written as

$$
s_{t c}(k, \theta)=A_{t} / A_{r} \cdot \operatorname{rect}\left(\frac{k-k_{c}}{B}\right) \cdot \exp \left(-j 2 k\left(R_{c}-R_{r}-(x \cos \theta+y \sin \theta)\right)\right)
$$

Generally, the echo signal of an arbitrary target rather than a point target is expressed by the following integral equation:

$$
G(k, \theta)=\iint_{x, y} F(x, y) \exp (j 2 k(x \cos \theta+y \sin \theta)) \exp \left(-j 2 k\left(R_{c}-R_{r}\right)\right) d x d y
$$


and the imaging process is presented by

$$
\hat{F}(x, y)=\iint_{k, \theta} G(k, \theta) \exp (-j 2 k(x \cos \theta+y \sin \theta)) \exp \left(j 2 k\left(\hat{R}_{c}-R_{r}\right)\right) k d k d \theta
$$

where $\hat{R}_{c}$ is the estimation of $R_{c}$ as $R_{c}$ is unknown. It can be seen from Eq. (9) that imaging is essentially the inverse process of the echo signal generation. The quality of an image is determined by the accuracy of the echo signal model and the matching degree of amplitude and phase accumulation compared to the echo signals. The main differences between Eqs. (8) and (9) and the familiar standard spatial Fourier transform pairs are the terms $\exp \left(-j 2 k\left(R_{c}-R_{r}\right)\right)$ and $\exp \left(j 2 k\left(\hat{R}_{c}-R_{r}\right)\right)$ in the integrant functions. The primary conclusion of the derivation from Eqs. (1) to (7) is the appearance of an extra term $\exp \left(-j 2 k\left(R_{c}-R_{r}\right)\right)$ in Eq. (8) compared to the standard Fourier transform; accordingly, a conjugate term $\exp \left(j 2 k\left(\hat{R}_{c}-R_{r}\right)\right)$ is involved in Eq. (9). It is this extra term that leads to a degradation of image quality in large-rotation angle cases, and our first part of work is developed essentially based on it.

\subsection{Influence Analysis}

In order to get an appropriate estimation of $R_{c}$ to correct the deviation distance $R_{c}-R_{r}$, entropy is introduced to evaluate the focusing quality of the THz images. Entropy has been successfully applied to evaluate the quality of SAR or ISAR images [26, 27]. The definition of entropy is [28]

$$
\mathrm{En}=\iint-f(x, y) \cdot \ln f(x, y) d x d y, f(x, y)=\frac{|F(x, y)|^{2}}{\iint|F(x, y)|^{2} d x d y}
$$

where $f(x, y)$ is the normalized image power density.

We use point spread function (PSF) to analyze the focusing quality of $\mathrm{THz}$ images utilizing the criterion given by Eq. (10). Suppose there is a point target located at the origin of the coordinate and set $\Delta R=R_{c}-R_{r}, \Delta \hat{R}=\hat{R}_{c}-R_{r}$ according to Eq. (9). Then, the expression of PSF is

$$
P S f(r, \phi)=\int_{\theta_{\min }}^{\theta_{\max }} \int_{k_{\min }}^{k_{\max }} \exp (-j 2 k(r \cos (\theta-\phi)-(\Delta \hat{R}-\Delta R))) k d k d \theta
$$

Particularly, when $\Delta \hat{R}-\Delta R=0$ and $\theta_{\max }-\theta_{\min }=2 \pi$, Eq. (11) has a compact form

$$
P s f(r, \phi)=k_{\max } \frac{J_{1}\left(2 k_{\max } r\right)}{\pi r}-k_{\min } \frac{J_{1}\left(2 k_{\min } r\right)}{\pi r}
$$

PSF is now an isotropic function of $r$. But in general cases, Eq. (11) has no compact form and PSF is a function of both $r$ and $\phi$. PSF can neither be expressed as a separable function of range and azimuth directions nor an isotropic function of $r$. Numerical simulations are employed to study the effects caused by the deviation distance and the rotation angle to the focusing quality. 
The simulation frequency ranges from 320 to $330 \mathrm{GHz}$, and the results are shown in Fig. 2. We can see that the colored scale is approximately limited to the range 4.5-7.5. This value is related to the number of pixels of the discretized image. The size of the image area in the simulation is $0.4 \mathrm{~m} \times 0.4 \mathrm{~m}$, and it is discretized into $250 \times 250$ pixels. As a digital image is made up of discrete grids, Eq. (10) should be discretized as

$$
\text { En }=-\sum_{n=1}^{N} \sum_{m=1}^{M} f(n, m) \cdot \ln f(n, m), f(n, m)=\frac{|F(n, m)|^{2}}{\sum_{n=1}^{N} \sum_{m=1}^{M}|F(n, m)|^{2}}
$$

where $n$ and $m$ represent the position of the pixel and $N$ and $M$ represent the total number of pixels in each row and each column. When entropy is treated as a criterion of image assessment, its physical meaning is that the more uniform the value of all the pixels, the higher the entropy is. For images with the same number of pixels, the one which all the pixels share the same value is of the highest entropy. For images whose pixels share the same value, the more the pixels, the higher the entropy is. Hence, the number of pixels in the image should be fixed when utilizing entropy as an assessment criterion. It is not hard to validate $\sum_{n=1}^{N} \sum_{m=1}^{M} f(n, m)=1$ in Eq. (13); consequently, $f(n, m)$ can be regarded as a probability distribution function and the entropy can be interpreted from the perspective of information theory. Suppose $f(n, m)$ stands for the probability distribution function of a random variable and then the colored value ranging from 4.5 to 7.5 in Fig. 2 represents the average information content of this random variable. Since natural logarithm function is utilized in Eq. (13), the unit of the colored value should be "nat" (natural unit).

The range of deviation distance and rotation angle in Fig. 2 is $\Delta \hat{R}-\Delta R \in$ $(-0.15 \mathrm{~m}, 0.15 \mathrm{~m})$ and $\theta_{\max }-\theta_{\min } \in\left(0^{\circ}, 60^{\circ}\right)$, respectively. It can be seen that for a fixed imaging rotation angle, PSF entropy always gets its minimum when the deviation distance is zero. Thus, it is reasonable to choose entropy as a criterion of the image quality. When the rotation angle is small (i.e., $<4^{\circ}$ approximately), the entropy of PSF decreases with the increasing of rotation angle which indicates that the accumulation of rotation angle makes the PSF sharper. Meanwhile, the large variation of deviation distance does not bring an

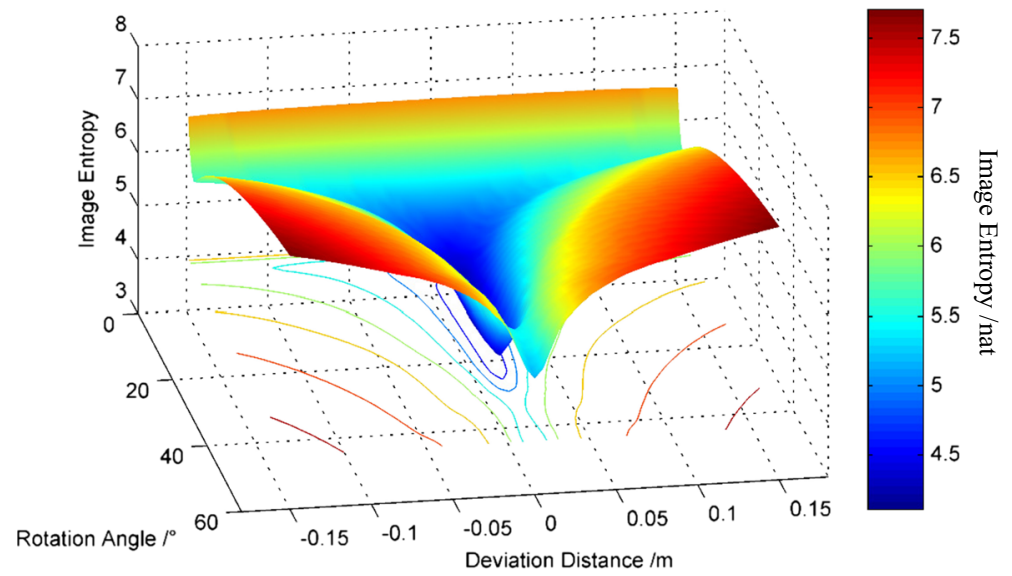

Fig. 2 The curve of the entropy of PSF varies against deviation distance and rotation angle 
obvious difference of the entropy. It is because the phase errors aroused by deviation distance are not critical and the coherence of echo signal maintains in azimuth direction in small-rotation angle cases. With the increasing of the rotation angle, the dependence of PSF entropy against deviation distance is strengthened and the entropy curve is relatively sharper where $\Delta \hat{R}-\Delta R$ approaches to zero. This feature contributes to the improvement of the estimation accuracy of the deviation distance. Besides, it can be found that the entropy does not decrease monotonically with the increasing of rotation angle when the deviation distance is zero. It is because PSF is obtained directly from Eq. (11) and no sidelobe suppression technology is utilized. We does not attempt to discuss this nonmonotonically decreasing problem in detail since it does not damage the entropy getting its minimum as the deviation distance approaches to zero.

As can be seen in Fig. 2, for a fixed rotation angle, image entropy always gets its minimum when the deviation distance is zero. It means that Eqs. (8) and (9) are completely matched/ focused as entropy gets its minimum in this ideal point scattering situation. However, whether it is true for complicated targets is still not verified by mathematical derivation. Besides minimum entropy algorithm (MEA), maximum contrast algorithm (MCA) is also a frequently used method. While MCA cares about the dominant scatters too much that most scatters may not be so well focused as the dominant one, MEA avoids this problem and it can attain a good compromise among all the scatters and results in a globally good image. The imaging results in the following measurement part also verify this point.

\subsection{Estimation of the Deviation Distance}

To estimate the deviation distance, a typical single-parameter optimization algorithm is employed as shown in Fig. 3.

\section{Analysis on Plane-wave Criteria in THz Imaging}

\subsection{Formulas of the Problem}

One can note that plane-wave approximation was introduced in the above derivation for convenience. However, it is more appropriate to depict the wavefront using Green's function rather than plane-wave function due to the limit of propagation distance and the short wavelength of the THz wave. The differences can be easily found in Fig. 4. From Fig. 4, we can conclude that whether a spherical wave can be approximated by a plane wave relies on three factors: the distance between the target and radar, the size of the target, and the wavelength.

Strictly speaking, Eq. (6) should be rewritten as

$$
R_{t}=\sqrt{r_{t}^{2}+R_{c}^{2}-2 r_{t} R_{c} \cos (\theta-\phi)}
$$

and the echo signal ought to be

$$
G(k, \theta)=\iint_{x, y} F(x, y) \exp \left(j 2 k\left(R_{c}-R_{t}\right)\right) \exp \left(-j 2 k\left(R_{c}-R_{r}\right)\right) d x d y
$$




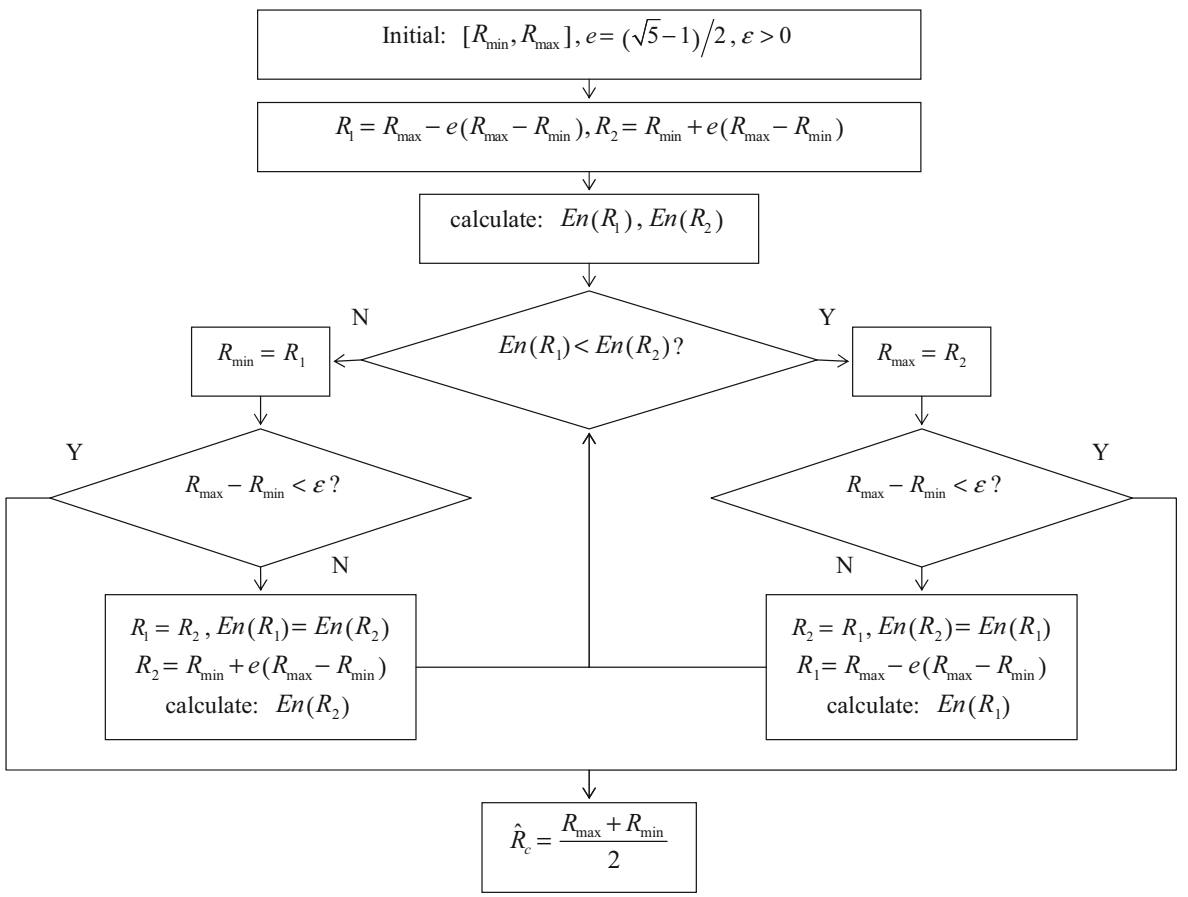

Fig. 3 Optimization flowchart

Consistently, the imaging formula is

$$
F(x, y)=\iint_{k, \theta} G(k, \theta) \exp \left(-j 2 k\left(\hat{R}_{c}-R_{t}\right)\right) \exp \left(j 2 k\left(\hat{R}_{c}-R_{r}\right)\right) k d k d \theta
$$

(a)

(b)
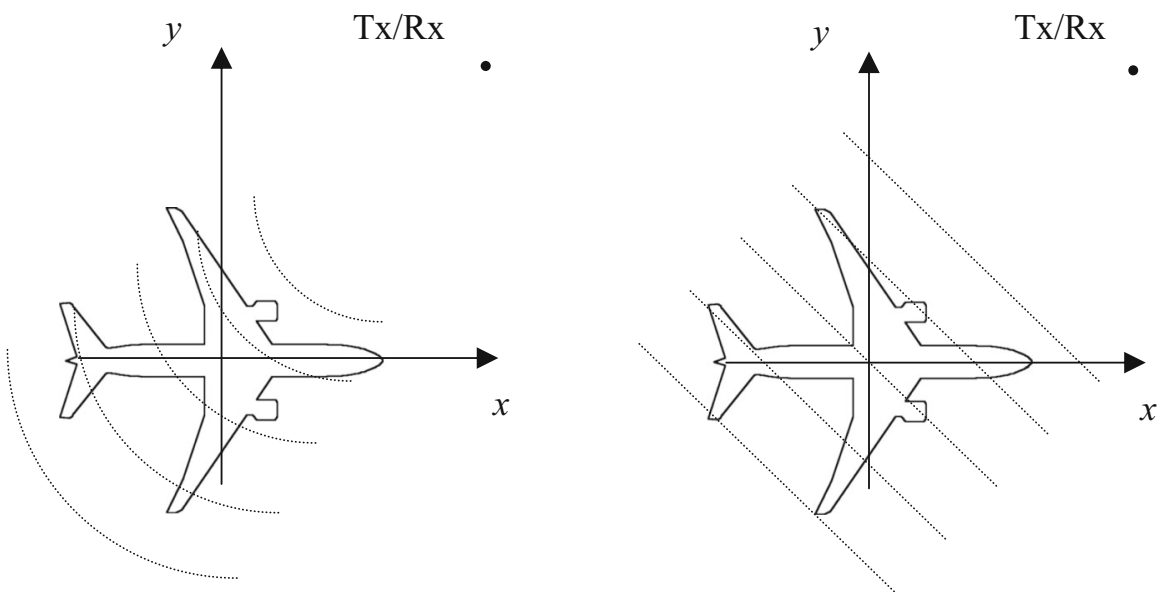

Fig. 4 A comparison of different illuminations between a spherical wave and $\mathbf{b}$ plane wave 
Set a point target $\left(x_{t}, y_{t}\right)=\left(r_{t} \cos \phi, r_{t} \sin \phi\right)$ and assume the deviation distance has been completely corrected which means $\hat{R}_{c}=R_{c}$. If we still use Eq. (9) rather than Eq. (16) to achieve images from the echo signal given by Eq. (15), then PSF is

$$
\operatorname{PSF}(x, y)=\iint_{k, \theta} \exp \left(j 2 k\left(R_{c}-R_{t}\right)\right) \exp (-j 2 k(x \cos \theta+y \sin \theta)) k d k d \theta
$$

Perform Taylor expansion on $R_{t}$ according to Eq. (14)

$$
R_{t}=R_{c}-r_{t} \cos (\theta-\phi)+\frac{r_{t}^{2}}{2 R_{c}} \sin ^{2}(\theta-\phi)+o\left(R_{c}^{-1}\right)
$$

and rewrite Eq. (17) using the expansion in Eq. (18) neglecting those higher-older terms

$$
\operatorname{PSF}(x, y)=\iint_{k, \theta} \exp \left(j 2 k\left(r_{t} \cos (\theta-\phi)-\frac{r_{t}^{2}}{2 R_{c}} \sin ^{2}(\theta-\phi)\right)\right) \exp (-j 2 k(x \cos \theta+y \sin \theta)) k d k d \theta
$$

We can learn from a previous analysis that the essential of imaging is a process of matching and accumulating amplitude and phase in the echo signal. The matching degree directly determines the quality of images. The unmatched phase term in Eq. (19) is

$$
\Phi_{e}=-j 2 k \frac{r_{t}^{2}}{2 R_{c}} \sin ^{2}(\theta-\phi)
$$

\subsection{Plane-wave Criterion in Small Rotation Angle Cases}

For the purpose of explaining the impacts of phase errors on images, we assume $\theta$ in Eq. (20) is minor enough. The reasonability of this assumption is based on two aspects: firstly, according to Eq. (19), imaging is a process of integrating upon rotation angle which can be decomposed into a number of small-rotation angle integration. Secondly, for arbitrary $\theta$, a small value of $\theta$ is obtained by rotating the target coordinate to make the $x$-axis pointing towards the radar. As the imaging process of arbitrary range of $\theta$ can be treat as a number of imaging processes of $\Delta \theta$, we can make following approximations:

$$
\left\{\begin{array}{l}
\sin \Delta \theta \approx \Delta \theta \\
\sin ^{2} \Delta \theta \approx \Delta \theta^{2} \\
\cos \Delta \theta \approx 1-0.5 \Delta \theta^{2} \\
\cos ^{2} \Delta \theta \approx 1-\Delta \theta^{2}
\end{array},\left\{\begin{array}{l}
k_{x}=k \cos \Delta \theta \approx k \\
k_{y}=k \sin \Delta \theta \approx k \Delta \theta
\end{array}\right.\right.
$$

Therefore, the $\Phi_{e}$ in Eq. (20) is approximated to [25]

$$
\Phi_{e}=-j \frac{x_{t}^{2}-y_{t}^{2}}{R_{c} k} k_{y}^{2}+j \frac{2 x_{t} y_{t}}{R_{c}} k_{y}-j \frac{y_{t}^{2}}{R_{c}} k_{x}
$$

Although looser criteria may exist, we define a strict quantitative criterion to judge whether $\Delta \theta$ is minor enough by supposing no migration range arises

$$
\Delta \theta<\frac{c}{B D}
$$


where $D$ is the diameter of the circumcircle of the target. According to Eq. (22), if we still generate images employing plane-wave approximation rather than Green's function, the extra phase term will bring two impacts: one is a position error of $-\frac{y_{t}^{2}}{2 R_{c}}$ in the range direction and $\frac{x_{t} y_{t}}{R_{c}}$ in the azimuth direction, respectively, and another is a focus-destroying phase term of $-\frac{x_{t}^{2}-y_{t}^{2}}{R_{c} k} k_{y}^{2}$. To guarantee the quality of focus, set $\left|\frac{x_{t}^{2}-y_{t}^{2}}{R_{c} k} k_{y}^{2}\right| \leq \frac{\pi}{8}$ (detailed introductions about this empirical value $\pi / 8$ can be found in [29]). Then, the plane-wave criterion in small-rotation angle cases is

$$
D \leq \frac{1}{2 \Delta \theta} \sqrt{R_{c} \lambda}
$$

The criterion defined by Eq. (24) for imaging is much looser than the criterion of the planewave approximation of spherical wave $D \leq \sqrt{R_{c} \lambda} / 2$. It is because the focusing quality relies on the relative variation of the phase rather than the absolute value of the phase. Thus, the difference of phase variation may be neglected although there are obvious absolute phase errors at some distance. Even though the absolute phase errors lead to position distortion of images, the focusing quality maintains when the rotation angle is small.

Figure $5(\mathrm{a}, \mathrm{b})$ shows the distortion of an $1 \mathrm{~m} \times 1 \mathrm{~m}$ image area under the condition of $R_{c}=2.5 \mathrm{~m}, \theta=0^{\circ}$, and $\theta=30^{\circ}$. The beginnings of the arrows indicate the real position of the pixels. The direction of the arrows represents the deviation direction of the pixels, and the length of the arrows is proportional to the degree of distortion.

\subsection{Plane-wave Criterion in Large Rotation Angle Cases}

In large-rotation angle cases, the process of imaging integration can be decomposed into a number of small-rotation angle integration and the image obtained by each small-rotation angle can achieve good focusing quality. But the orientation and distance of the distortion are both different as a result of different observation angles. After summing up all these sub-images, a point target may be mapped to a distorted curve in the image. Figure 5 illuminates this problem that the same point has different distortions by comparing Fig. 5(a)

(a)

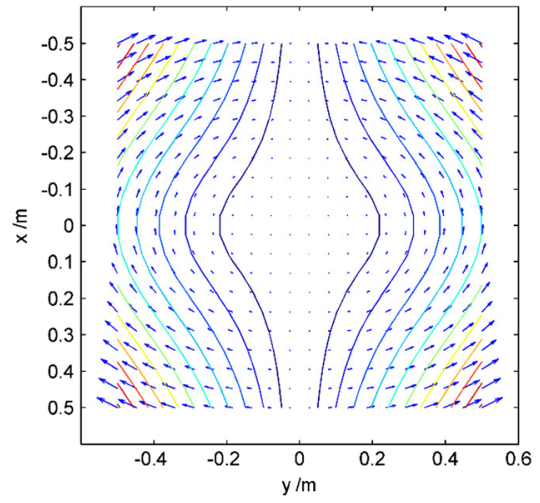

(b)

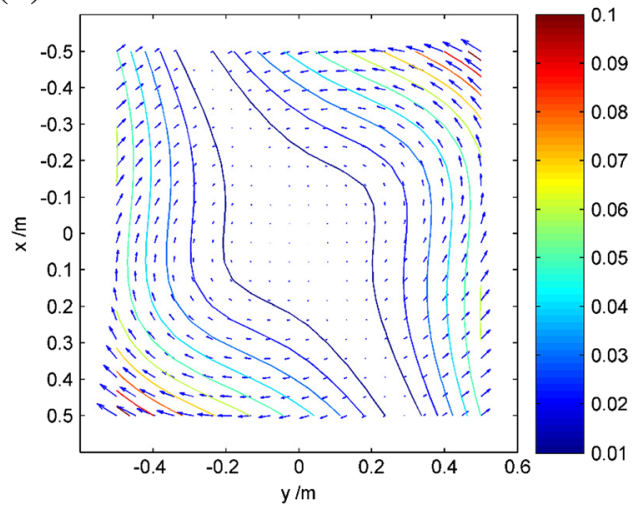

Fig. 5 This figure illuminates the position distortion of the image. The beginning arrows stand for the real position of the pixels, and the ending arrows represent the distorted positions of the pixels: $a$ position distortion with observation angle $\theta=0^{\circ}$ and $b$ position distortion with observation angle $\theta=30^{\circ}$ 
with Fig. 5(b). Figure 6 shows this problem concisely. The beginning of the arrows indicates the distorted position of the pixels when $\theta=0^{\circ}$. The ending of the arrows indicates the distorted position of the pixels when $\theta=30^{\circ}$.

Now, we will derive the analytic form of the plane-wave criterion in large-rotation angle cases. Utilize the results derived in small-rotation angle cases and suppose $\theta=0^{\circ}$. Then, the position distortion is

$$
\left.d x\right|_{\theta=0}=-\frac{y^{2}}{2 R_{c}} \quad,\left.d y\right|_{\theta=0}=\frac{x y}{R_{c}}
$$

If $\theta \neq 0^{\circ}$, apply coordinate transforms $u=x \cos \theta+y \sin \theta$ and $v=-x \sin \theta+y \cos \theta$ and the distortion in a new coordinate is

$$
d u=-\frac{u^{2}}{2 R_{c}}, d v=\frac{u v}{R_{c}}
$$

Transforming Eq. (26) back into the original coordinate, we obtain

$$
\left.d x\right|_{\theta}=d u \cos \theta-d v \sin \theta,\left.\quad d y\right|_{\theta}=d u \sin \theta+d v \cos \theta
$$

According to Eqs. (25) and (27), the displacement of the pixel is

$$
\vec{s}=\left(\left.d x\right|_{\theta}-\left.d x\right|_{\theta=0},\left.d y\right|_{\theta}-\left.d y\right|_{\theta=0}\right)^{\mathrm{T}}
$$

To guarantee the focusing quality of images, the displacement of point targets is supposed to be confined within the resolution unit

$$
\vec{s}^{\mathrm{T}} \vec{s} \leq\left(\frac{c}{2 B}\right)^{2}+\left(\frac{\lambda}{4 \sin (\theta / 2)}\right)^{2}
$$

We have employed the definition of resolution from [30]. Equation (29) is the criterion of the plane-wave approximation in large-rotation angle cases.

Fig. 6 This figure shows the distortion when the imaging rotation angle is $30^{\circ}$. The beginning arrows stand for the distorted position when $\theta=0^{\circ}$, and the ending arrows represent the distorted position when $\theta=30^{\circ}$

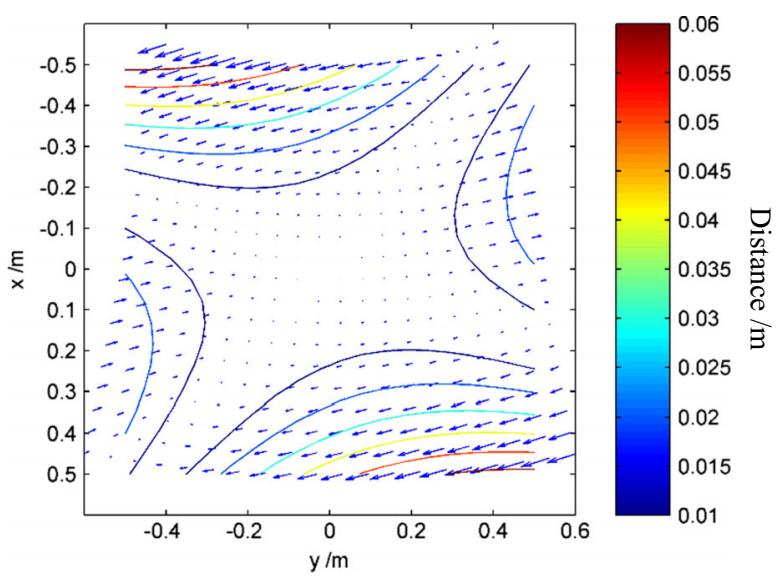




\section{Experimental Results and Analysis}

The parameters of the radar system and experimental configurations are listed in Table 1 and Fig. 7.

According to Eq. (23) and Table 1, the threshold separating small and large rotation angles is $4.3^{\circ}$ in our experiments. We choose $2^{\circ}$ and $30^{\circ}$ as representatives of small and large rotation angles, respectively. Setting $\Delta \theta=2^{\circ}$ in Eq. (24), we can obtain the condition of plane-wave approximation that is $D \leq 0.68 \mathrm{~m}$. Similarly, setting $\theta=30^{\circ}$ in Eq. (29), we obtain $D \leq 0.61 \mathrm{~m}$. As the diameter of the circumcircle of targets is less than $0.5 \mathrm{~m}$, we predict that plane-wave approximation is still valid under our experimental configurations.

\subsection{Imaging Results of Corner Reflectors}

It is easy to find Fig. 8(a) shares a great resemblance to Fig. 8(b). The only difference is the displacement in the range direction ( $x$-axis) which is a result of the compensation of the deviation distance. The sidelobes are apparent as no suppression technology is employed. The resolution is not outstanding as only a small rotation angle is utilized. It is necessary to claim that the image area is discretized into $400 \times 400$ pixels and the image entropy before and after the compensation is 8.41 and 8.40 , respectively. Just like what the image entropy indicates, no remarkable changes are observed after the deviation compensation and it is consistent with what is shown in Fig. 2 in small-rotation angle regions.

As shown in Fig. 9, when the rotation angle increases to $30^{\circ}$, obvious differences are found between the images obtained before and after compensation. In Fig. 9(a), the quality of the image decreases instead of increasing with the increasing of the rotation angle. This phenomenon can be explained from two aspects. On the one hand, imaging is an essential procedure of 2D-matched filtering. The deviation between the center of turntable and the reference point leads to an unmatched filtering and results in the defocus of the image. The larger the rotation angle, the worse the focusing of the image is. On the other hand, large-rotation angle imaging can be decomposed into a number of small-rotation angle imaging. We can learn from Fig. 8 that no defocus happens in the image obtained by small rotation angle and the deviation distance $\Delta \hat{R}-\Delta R$ only leads to a shift in the range direction. When we transform all these subimages back to the original coordinate and sum them up, little arcuate curves will appear in the final image as shown in Fig. 9(a). Comparing Fig. 9(b) with Figs. 9(a) and 8(b), notable improvements of resolution and SNR can be easily achieved by increasing the rotation angle after the deviation compensation. Quantitatively, the image entropy of Fig. 9(a) is 9.31 and that of Fig. 9(b) is 6.69, which also indicates a greater promotion of image quality than that of Fig. 8. This phenomenon is highly consistent with and can be predicted by Fig. 2.

Table 1 Parameters of 0.33-THz LFMCW radar and experimental configurations

\begin{tabular}{ll}
\hline Central frequency & $330 \mathrm{GHz}$ \\
Bandwidth & $8 \mathrm{GHz}$ \\
Pulse duration & $300 \mu \mathrm{s}$ \\
Total rotation angle & $33^{\circ}$ \\
Distance between radar and the turntable center & $2.5 \mathrm{~m}$ \\
Size of an airplane model & $0.5 \mathrm{~m} \times 0.5 \mathrm{~m}$ \\
\hline
\end{tabular}



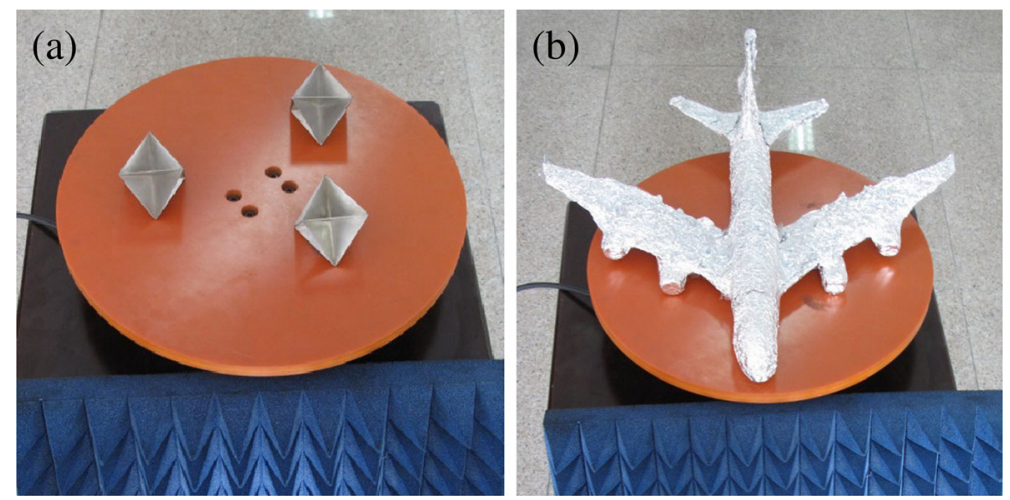

Fig. 7 Photos of a corner reflectors and $\mathbf{b}$ the aircraft model on a circular turntable

Now, we discuss the effects that spherical wave brings to the image. We utilize Eqs. (9) and (16) to generate images using the measured echo data, respectively. For convenience of comparing the difference, we plot these two images in the same figure as shown in Fig. 10(a) and a partial zoomed in figure is plotted in Fig. 10(b).

In Fig. 10, the images obtained utilizing Eqs. (9) and (16) show no apparent difference but slight position displacements. It is because the distance between corner reflectors and the turntable center is relatively small. Figure 10(b) shows the detailed position of the same corner obtained by Eqs. (9) and (16), respectively.

\subsection{Imaging Results of the Aircraft Model}

Similar with Fig. 8, the only apparent difference between (a) and (b) in Fig. 8 is a shift in the range direction ( $x$-axis). It is necessary to claim that the image area is discretized into $360 \times 312$ pixels and the image entropy before and after the compensation is 9.38 and 9.37 , respectively. The deviation compensation does not lead to a notable improvement of the image quality. This phenomenon can also be explained by Fig. 2. Due to the limitation of small rotation angle, the image resolution is limited and only a coarse contour of the aircraft model emerges. Also, in this case, we can see that the image suffers from low SNR since an aircraft model is not a target as bright as a corner reflector.

(a)

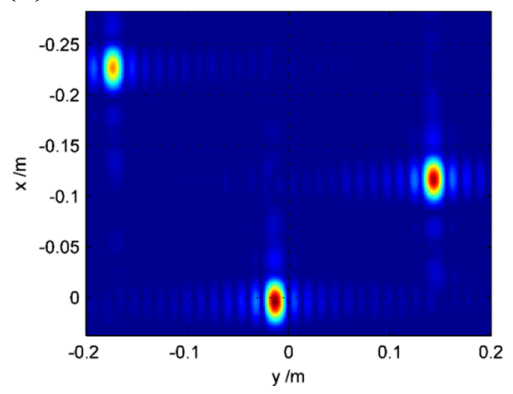

(b)

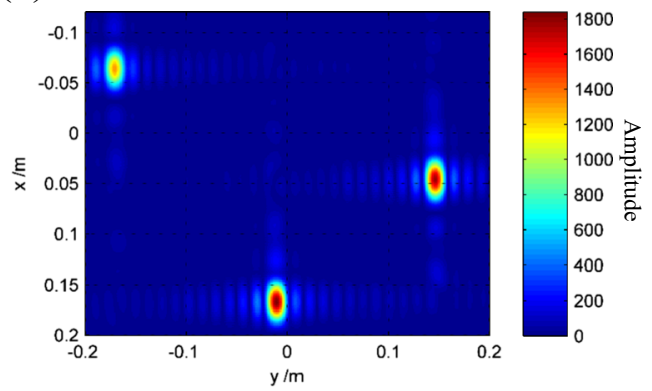

Fig. 8 Images of corner reflectors with the rotation angle from $\theta=0^{\circ}$ to $\theta=2^{\circ}$ under plane-wave approximation $a$ before and $b$ after the deviation distance compensation 
(a)

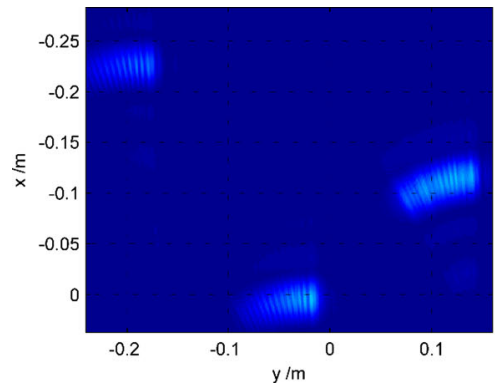

(b)

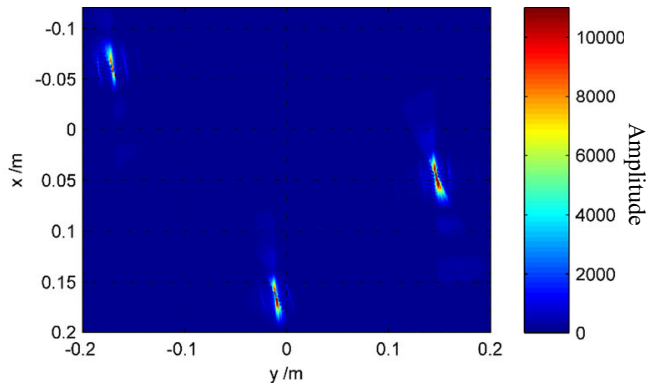

Fig. 9 Images of corner reflectors with the rotation angle from $\theta=0^{\circ}$ to $\theta=30^{\circ}$ under plane-wave approximation $a$ before and $b$ after the deviation distance compensation

Similar with Fig. 9, due to the deviation distance between the turntable center and the reference point, the image quality degrades instead of upgrading dramatically with the increasing of the rotation angle. Comparing Fig. 12(a) with Fig. 9(a), both images show serious defocus and one difference is that no arcuate defocus curves are found in Fig. 12(a) which is an interesting characteristic in Fig. 9(a). It is because there are denser scattering centers in the aircraft image and the scattering component cannot be described by scattering centers existed in the echo signal. Comparing Fig. 12(b) with Figs. 12(a) and 11(b), a large rotation angle paralleled by deviation compensation brings a remarkable improvement of the resolution and SNR. The head, turbines, wings, tail, and other details of the aircraft model can be clearly distinguished in Fig. 12(b). Quantitatively, the image entropy of Fig. 12(a) is 9.54 and that of Fig. 12(b) is 9.03. In addition, an arc-shaped ribbon can be observed near the head of the aircraft. It is attributed to the migrator scattering from the edge of the circular turntable. This phenomenon also exists in Fig. 9(b), but it is suppressed by the bright corner scattering. The arc-shaped ribbon will emerge by adopting logarithm magnitude and adjusting the dynamic range of the image. We do not attempt to implement further discussions about this phenomenon in this paper.

Figure 13(a, b) shows the images obtained utilizing Eqs. (9) and (16), respectively. It is hard to distinguish their difference unless painstaking comparison is carried out. This is a result of a

(a)

(b)
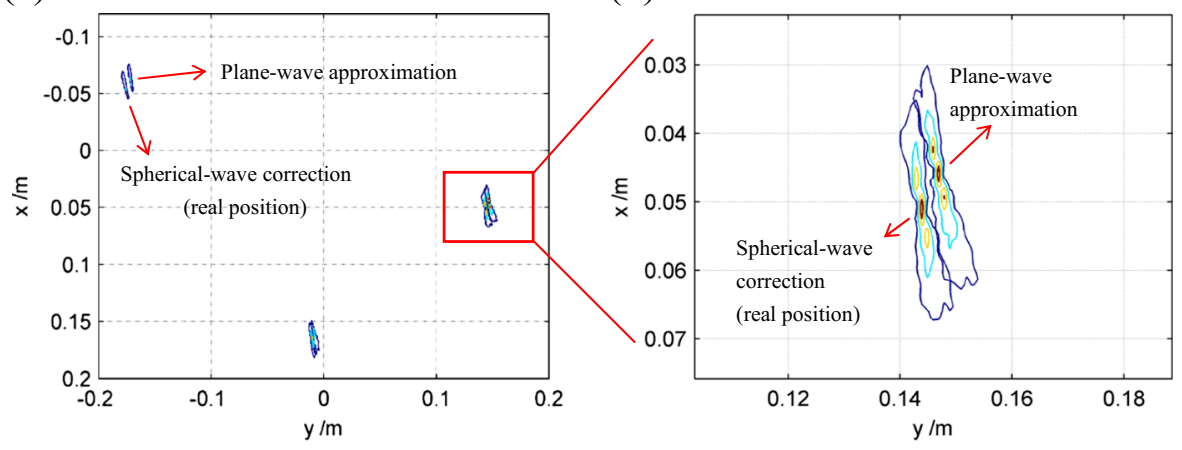

Fig. 10 Images of corner reflectors with the rotation angle from $\theta=0^{\circ}$ to $\theta=30^{\circ}$ after the deviation compensation. $a$ Images obtained by Eqs. (9) and (16) superposed in one coordinate. $b$ A partial zoomed in figure of one corner reflector 
(a)

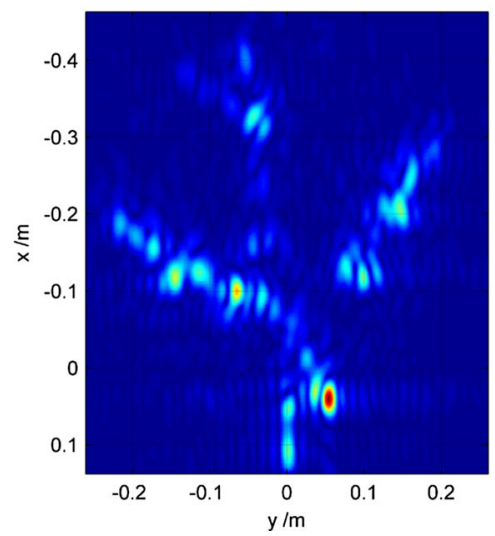

(b)

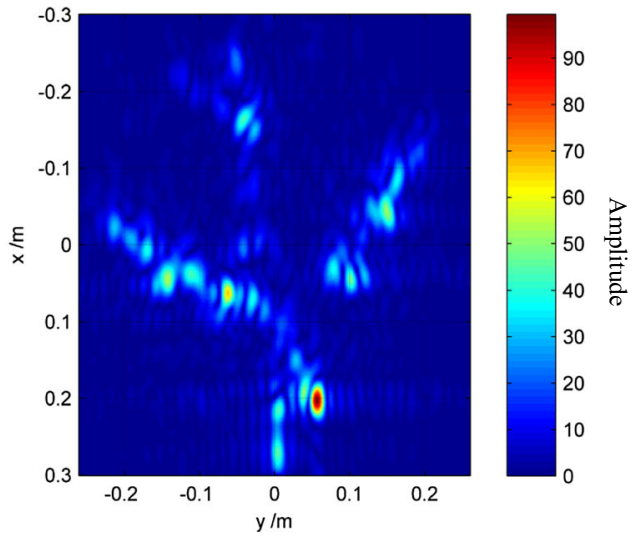

Fig. 11 Images of the aircraft model with the rotation angle from $\theta=0^{\circ}$ to $\theta=2^{\circ}$ under plane-wave approximation $a$ before and $b$ after the deviation distance compensation

relatively small size of the model, and it can also be predicted by Eq. (29) as shown in Section 3. Comparing the highlighted region in the circles, a slight distortion of spherical wave can be noticed from the bent wings and the constricted tails.

\subsection{Analysis and Discussion}

More quantitative analyses on the image entropy, plane-wave approximation, and spatial resolution are carried out to show the effectiveness and correctness of our proposed method and derivation. Then, the pros and cons of this method for potential applications are discussed.

Figure 14 illustrates the image entropy of corner reflectors and the aircraft model before/ after the deviation compensation varies against rotation angle. As we can see, both solid lines are obviously lower than the dashed ones for most range of the rotation angle, which is the

(a)

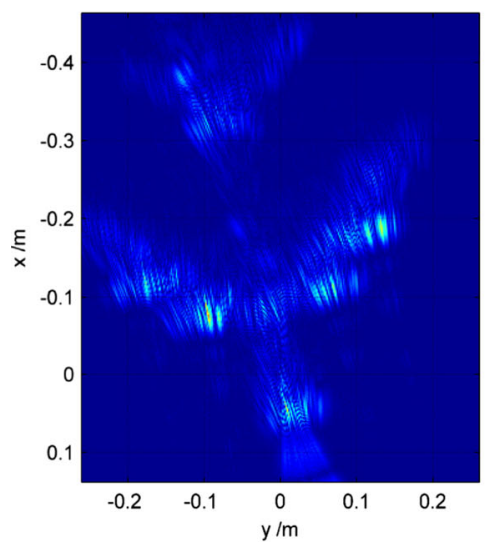

(b)

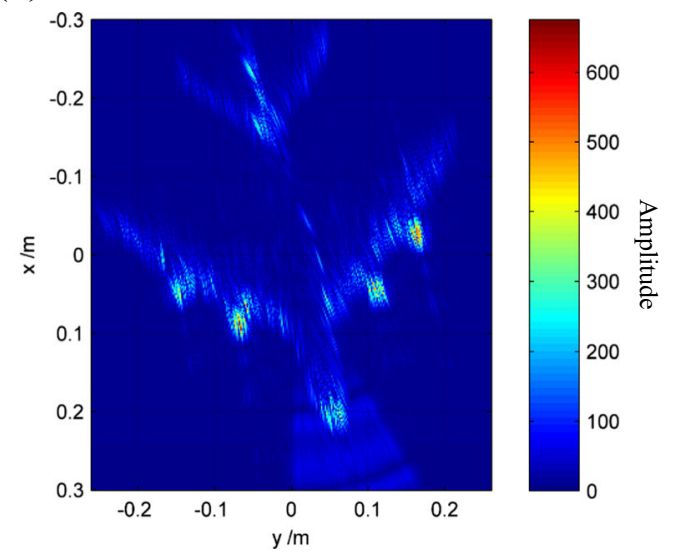

Fig. 12 Images of the aircraft model with the rotation angle from $\theta=0^{\circ}$ to $\theta=30^{\circ}$ under plane-wave approximation $a$ before and $b$ after the deviation distance compensation 
(a)

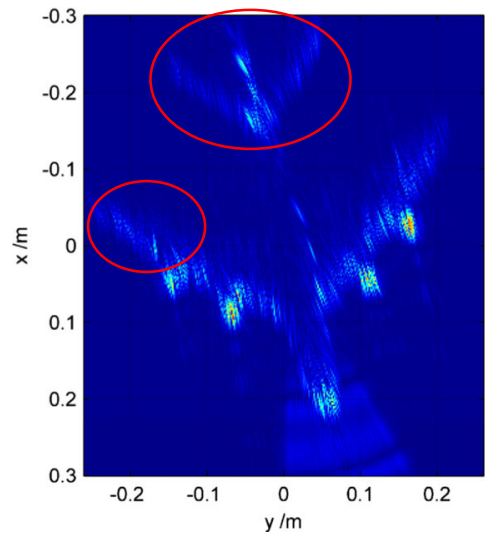

(b)

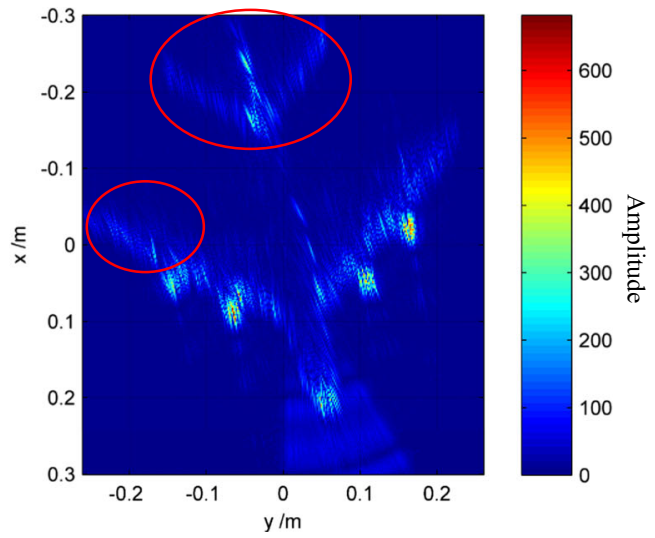

Fig. 13 Images of the aircraft model with the rotation angle from $\theta=0^{\circ}$ to $\theta=30^{\circ}$ after the deviation compensation results $a$ using Eq. (9) under plane-wave approximation and $b$ using Eq. (16) considering the spherical-wave effects

purpose of the deviation compensation. In Fig. 14a, both lines drop rapidly from 11 to 8 as the rotation angle increases from $0^{\circ}$ to about $3^{\circ}$. From this point onwards, the image entropy after the deviation compensation declines consecutively while the declining rate becomes lower and lower. In contrary, the dashed line which stands for the image entropy before deviation compensation goes upward after the rotation angle exceeds $3^{\circ}$ and keeps ascending as the rotation angle grows larger. These phenomena revealed by experiments show high consistency with what is shown in Fig. 2, achieved by simulation. In Fig. 14b, when the rotation angle is smaller than $7^{\circ}$, the entropy before and after the deviation compensation is very similar and they both experience a bumpy descend. In the region where a rotation angle is larger than $7^{\circ}$, the solid and dashed lines show an apparent difference. The dashed line firstly sees a slight jump followed by a steady moderate rise, while the solid line keeps sinking at first, bottoming out at around $15^{\circ}$. After that, it also experiences a steady moderate grow just like the dashed line does.

(a)

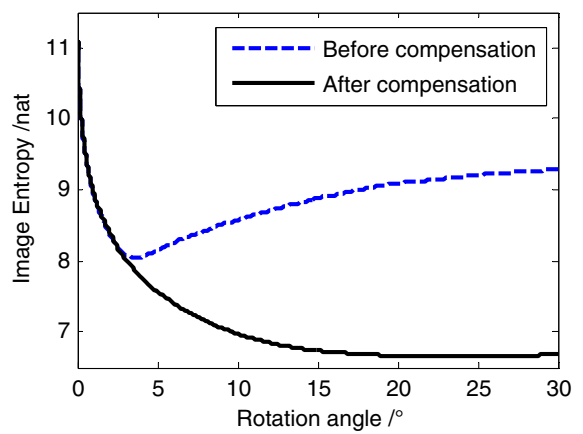

(b)

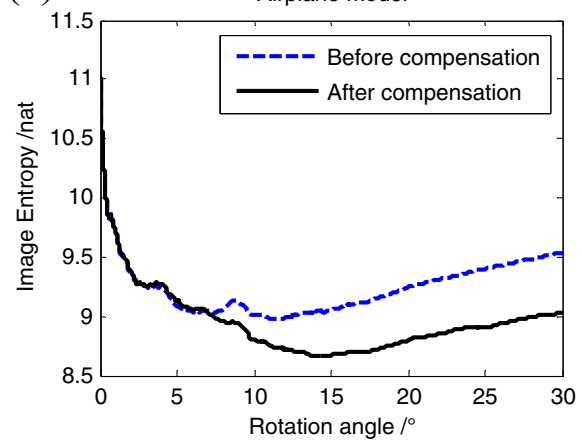

Fig. 14 Line graph of image entropy before/after deviation compensation varies against rotation angle. a Image entropy of the corner reflectors. b Image entropy of the aircraft model 
Comparing Fig. 14b with Fig. 14a, we can find three main differences: firstly, the lines in (b) is not as smooth as those in (a); secondly, the distance between the solid line and the dashed line in (b) is smaller than that of (a), especially in large-rotation angle cases; thirdly, the solid line in (b) sees an obvious grow while that one in (a) does not. Different from corner reflectors which can be treated as ideal point reflectors in a wide range of observation angle, an aircraft model may stimulate a variety of scattering components. As previously mentioned, the migrator scattering from the edge of the circular turntable which leads to an arc-shaped ribbon in the image of the aircraft model as shown in Figs. 12(b) and 13 can make great contribution to raise the image entropy. Apart from migrator scattering, the effects of surface roughness of the model to the images will also promote the image entropy as the resolution is improved by a large rotation angle. This nonmonotonic trend of the curve can challenge the reasonability of using entropy as a criterion of the image quality because it goes against that larger rotation angle can capture more information and will lead to better image quality. This is a problem that needs further research. Nonetheless, it is necessary to clarify that the nonmonotonicity does not affect the proposed minimum entropy-based autofocus method for a fixed rotation angle.

Considering the complicated scattering components contained in the scattered wave of aircraft model, it is difficult to conduct a concise analysis on the image distortion caused by plane-wave approximation using Fig. 13. Thus, Fig. 10, the image of corner reflectors, is chosen to probe the influence of plane-wave approximation quantitatively. In Fig. 10(b), the coordinate of the lower-left peak is $(0.051,0.144)$ and that of the upper-right peak is $(0.046$, $0.147)$. In the upper-left corner of Fig. 10(a), the coordinate of the left peak is $(-0.053,-0.176)$ and that of the upper-right peak is $(-0.058,-0.171)$. According to the principles illustrated by Figs. 5 and 6, we can get the theoretically predicted distorted coordinates of the peaks $(0.051$, $0.144)$ and $(-0.053,-0.176)$ which are $(0.047,0.147)$ and $(-0.059,-0.172)$, respectively. As can be seen, the relative error of the distorted position between the analytical prediction and the experimental result is under $3 \%$ and the level of distortion is low enough to avoid errors when interpreting the image.

Figure 15 shows the PSF of a corner reflector and its cross section in range/azimuth direction to explore the image resolution experimentally. The corner reflector in Fig. 15 is the one in the right of Fig. 10(a). Strictly speaking, instead of measuring the resolution in the range and azimuth directions separately, a range-azimuth-coupled 2D resolution is more appropriate for image assessment when the rotation angle approaches $30^{\circ}$. Since this will be another topic out of the scope of this paper and for the convenience of comparison, the resolution here is still measured in range and azimuth directions separately. A new coordinate, range-to-azimuth coordinate, is established. The origin is situated at the peak of the PSF, and the range axis orients to radar at the middle observation angle which is determined by the geometry shown in Fig. 1 and the rotation angle. The cross section in range/azimuth direction is calculated and a $-3-\mathrm{dB}$ benchmark is used to measure the resolution. When the rotation angle is $2^{\circ}$, the measured range resolution is $2.30 \mathrm{~cm}$ and the azimuth is $1.16 \mathrm{~cm}$. When the rotation angle is $30^{\circ}$, the measured range resolution is $1.03 \mathrm{~cm}$ and the azimuth is under $0.08 \mathrm{~cm}$. Theoretically, we adopt the resolution definition in [30] in large-rotation angle cases. According to the parameters listed in Table 1, the range resolution is $1.72 \mathrm{~cm}$, the azimuth resolution under $2^{\circ}$ rotation angle is $1.32 \mathrm{~cm}$, and the azimuth resolution under $30^{\circ}$ rotation angle is under $0.09 \mathrm{~cm}$. Compared to the resolution measured by the experiments, we can find that the resolution estimation in [30] is a conservative one. Over all, we can see that after the deviation distance compensation, at least 10 times promotion of resolution is achieved by the increased rotation angle and the azimuth resolution has already reached a sub-millimeter level. 
(a)
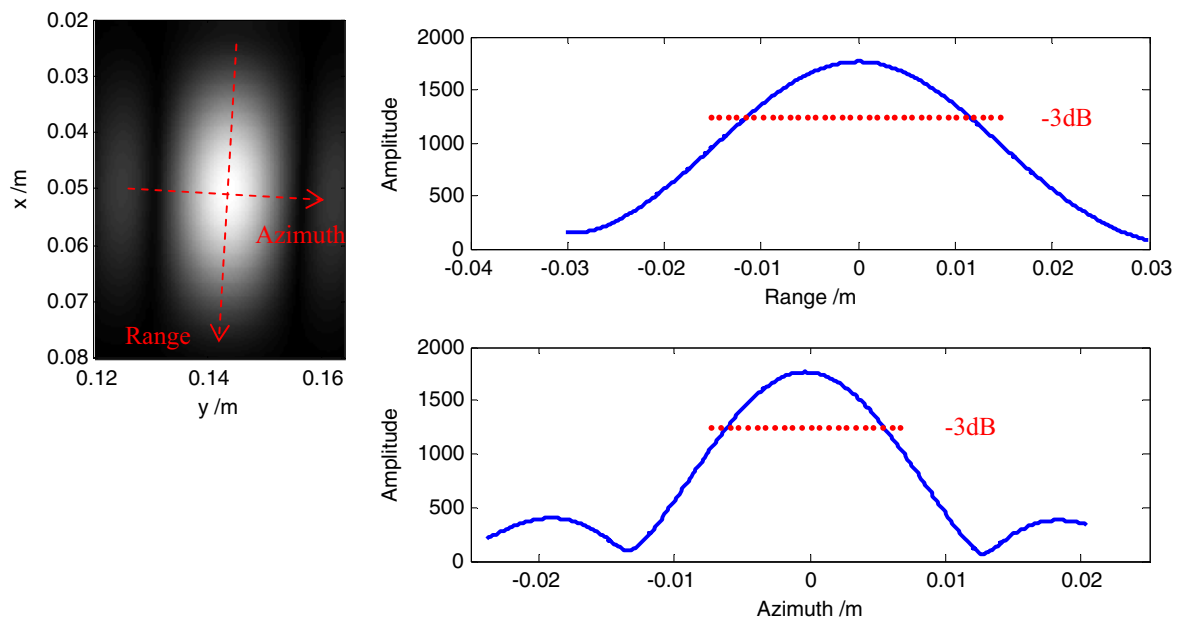

(b)
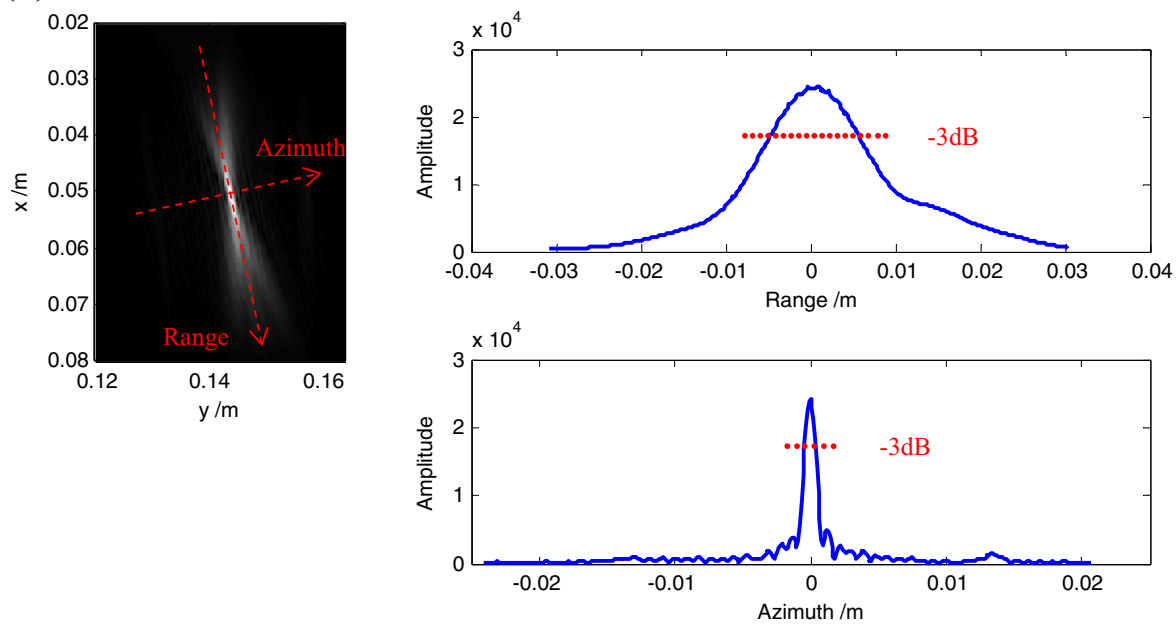

Fig. 15 Point spread function (PSF) and cross section in range/azimuth direction. a Rotation angle $=2^{\circ}$. b Rotation angle $=30^{\circ}$

Apart from synthetic aperture (SA) imaging, other popular imaging methods mainly include raster scanning method $[4,10]$ and focal plane array method [31]. Compared to a raster scanning method, no scanning motors or cumbersome reflectors are needed for SA imaging. Thus, the size of radar and the power requirements can be confined within a feasible scope. When compared to a focal plane array method, no careful quasi-optical setups are required for SA imaging. Recently, all that is needed in SA imaging is just a pair of transistor/receiver and a turntable. Besides, without the constraint of the Rayleigh limit of the spot size for raster scanning or the limited number of pixels in focal plane array, the resolution of SA image is theoretically independent with distance. It means that much sharper images can be obtained by SA techniques in terahertz regime. 
With the nonionizing nature and the capability to penetrate common packaging materials, $\mathrm{THz}$ wave has drawn much attention for applications such as personnel screening, noninvasive inspection, nondestructive evaluation, and biological applications. By using our improved algorithm, a general simple application scene can be conceived: with the target object positioned on a steadily running turntable and the radar system transiting and receiving $\mathrm{THz}$ waves, a sub-millimeter resolution image of the object is generated simultaneously. Although a centimeter resolution may be enough for identifying threats, such as knives and guns, a submillimeter resolution can still contribute to raise the detection rate and the veracity of identification. Also, evaluating the quality of tablets and detecting lymph nodes in human bodies are both potential applications of the improved SA imaging method.

However, some problems should still be considered carefully. Firstly, as aforementioned, real objects stimulate complex scattering components and this will add difficulty to image interpretation, especially if there are several layers. The THz beam may penetrate some layers, reflect between two layers, or be absorbed by some layers, and the obtained image can be seen rather different from the object. Therefore, additional studies to evaluate the scattering properties of a layered target and the needed dynamic range of the radar system must be performed. Secondly, the phase error discussed in this manuscript is under the assumption that the distance between radar and the target is a constant. It means that the target object is collaborating with the imaging process. More complex situations, such as safety inspection for walking bodies, can emerge in practical applications for uncooperative objects. As a result, parameters, such as the moving velocity of the target body, must be estimated to compensate phase errors caused by the movement before imaging. Much further research is needed to solve these problems.

\section{Conclusions}

Based on a 330-GHz LFMCW terahertz radar system, we presented two parts of work aiming at the large-rotation angle problem and the spherical-wave problem, respectively. On the one hand, we illustrated analytically that the deviation distance between the turntable center and the reference bright target leads to an extra linear phase term in the calibrated IF signal. In smallrotation angle cases, the quality of the image shows low dependence against this deviation distance and all the visible differences brought by this deviation are a shift of the image in the range direction. In large-rotation angle cases, the existence of deviation distance will bring dramatically degradation to the image quality. By utilizing the minimum entropy criterion to estimate and compensate for the phase errors, great improvements of resolution and SNR of the image were achieved. On the other hand, the criteria of plane-wave approximation for imaging have been analyzed and derived in small and large-rotation angle cases. Their compact form is expressed in Eqs. (24) and (29), respectively. These criteria are much looser than the criterion of the plane-wave approximation of spherical wave. When $R_{c}=2.5 \mathrm{~m}$ and the target size was smaller than $0.5 \mathrm{~m}$, experimental results showed that spherical wave only leads to a slight position distortion of images and the influence of spherical-wave effects on focusing quality can be neglected.

Acknowledgments This work was supported by the National Natural Science Foundation for Young Scientists of China (61302148) and the National Natural Science Foundation of China (61171133). The authors would also like to thank the anonymous reviewers for their valuable comments and suggestions to improve the paper quality. 
Open Access This article is distributed under the terms of the Creative Commons Attribution 4.0 International License (http://creativecommons.org/licenses/by/4.0/), which permits unrestricted use, distribution, and reproduction in any medium, provided you give appropriate credit to the original author(s) and the source, provide a link to the Creative Commons license, and indicate if changes were made.

\section{References}

1. N. Palka, R. Panowicz, F. Ospald and R. Beigang, "3D Non-destructive Imaging of Punctures in Polyethylene Composite Armor by THz Time Domain Spectroscopy," Journal of Infrared, Millimeter, and Terahertz Waves, vol. 36, no. 8, pp. 770-788, 2015.

2. A. J. Fitzgerald, E. Berry, N. N. Zinovev, G. C. Walker, M. A. Smith and J. M. Chamberlain, "An introduction to medical imaging with coherent terahertz frequency radiation," Physics in Medicine and Biology, vol. 47, no. 7, pp. R67-R84, 2002.

3. K. Ajito and Y. Ueno, "THz chemical imaging for biological applications," IEEE Transactions on Terahertz Science and Technology, vol. 1, no. 1, pp. 293-300, 2011.

4. K. B. Cooper, R. J. Dengler, N. Llombart, B. Thomas, G. Chattopadhyay and P. H. Siegel, "THz imaging radar for standoff personnel screening," IEEE Transactions on Terahertz Science and Technology, vol. 1, no. 1, pp. 169-182, 2011.

5. R. Appleby and H. B. Wallace, "Standoff detection of weapons and contraband in the $100 \mathrm{GHz}$ to $1 \mathrm{THz}$ region," IEEE transactions on antennas and propagation, vol. 55, no. 11, pp. 2944-2956, 2007.

6. K. B. Cooper, R. J. Dengler, N. Llombart, T. Bryllert, G. Chattopadhyay and E. Schlecht, et al., "Penetrating 3-D imaging at 4-and 25-m range using a submillimeter-wave radar," IEEE Transactions on Microwave Theory and Techniques, vol. 56, no. 12, pp. 2771-2778, 2008.

7. R. J. Dengler, F. Maiwald and P. H. Siegel, in Microwave Symposium Digest, 2006. IEEE MTT-S International, San Francisco, CA, 2006, pp. 1923-1926

8. R. J. Dengler, K. B. Cooper, G. Chattopadhyay, I. Mehdi, E. Schlecht and A. Skalare, et al., in Microwave Symposium, 2007. IEEE/MTT-S International, IEEE, Honolulu, HI, 2007, pp. 1371-1374

9. N. Llombart, K. B. Cooper, R. J. Dengler and P. H. Siegel, IEEE, 2010, pp. 1-4.

10. B. Blazquez, K. B. Cooper and N. Llombart, "Time-Delay Multiplexing With Linear Arrays of THz Radar Transceivers," IEEE Transactions on Terahertz Science and Technology, vol. 4, no. 2, pp. 232-239, 2014.

11. H. Essen, A. Wahlen, R. Sommer and W. Johannes, "High-bandwidth $220 \mathrm{GHz}$ experimental radar," Electronics Letters, vol. 43, no. 20, pp. 1114-1116, 2007.

12. S. Stanko, M. Caris, A. Wahlen, R. Sommer, J. Wilcke and A. Leuther, et al., in Millimeter Resolution with Radar at Lower Terahertz, 2013, pp. 235-238.

13. C. A. Weg, W. V. Spiegel, F. Senzel, R. Henneberger, R. Zimmermann and T. Loffler, et al., "Fast active THz-camera with global illumination," in Proc. 34th International Conference on Infrared, Millimeter, and Terahertz Waves. IRMMW-THz 2009, 2009, pp. 1-2.

14. D. M. Sheen, T. E. Hall, R. H. Severtsen, D. L. McMakin, B. K. Hatchell and P. L. J. Valdez, "Active wideband $350 \mathrm{GHz}$ imaging system for concealed-weapon detection," Proceedings of SPIE - The International Society for Optical Engineering, 2009

15. B. Kapilevich, Y. Pinhasi, R. Arusi, M. Anisimov, D. Hardon and B. Litvak, et al., " $330 \mathrm{GHz}$ FMCW Image Sensor for Homeland Security Applications," Journal of Infrared, Millimeter and Terahertz Waves, vol. 31, no. 11, pp. 1370-1381, 2010.

16. A. A. Danylov, T. M. Goyette, J. Waldman, M. J. Coulombe, A. J. Gatesman and R. H. Giles, et al., "Coherent imaging at $2.4 \mathrm{THz}$ with a CW quantum cascade laser transmitter," Proc SPIE, 2010.

17. M. Ravaro, V. Jagtap, G. Santarelli, C. Sirtori, L. Li and S. P. Khanna, et al., "Continuous-wave coherent imaging with terahertz quantum cascade lasers using electro-optic harmonic sampling," Applied Physics Letters, vol. 102, no. 9, pp. 91107, 2013

18. D. A. Robertson, S. L. Cassidy, B. Jones, A. Clark, D. A. Robertson and S. L. Cassidy, et al., in Society of Photo-Optical Instrumentation Engineers (SPIE) Conference Series, 2014, pp. 11084-11488.

19. M. Y. Liang, C. L. Zhang, R. Zhao and Y. J. Zhao, "Experimental 0.22 THz Stepped Frequency Radar System for ISAR Imaging," Journal of Infrared, Millimeter and Terahertz Waves, vol. 35, no. 9, pp. 780789, 2014.

20. C. Am Weg, W. von Spiegel, R. Henneberger, R. Zimmermann, T. Loeffler and H. G. Roskos, "Fast Active THz Cameras with Ranging Capabilities," Journal of Infrared, Millimeter and Terahertz Waves, 2009.

21. X. Gao, C. Li, S. Gu and G. Fang, "Design, Analysis and Measurement of a Millimeter Wave Antenna Suitable for Stand off Imaging at Checkpoints," Journal of Infrared, Millimeter and Terahertz Waves, vol. 32, no. 11, pp. 1314-1327, 2011. 
22. N. E. Alexander, B. Alderman, F. Allona, P. Frijlink, R. Gonzalo and M. Hägelen, et al., "TeraSCREEN: multi-frequency multi-mode Terahertz screening for border checks," Society of Photo-optical Instrumentation Engineers Conference Series, vol. 9078, no. 2, pp. 131-137, 2014.

23. B. Cheng, G. Jiang, C. Wang, C. Yang, Y. Cai and Q. Chen, et al., "Real-Time Imaging With a $140 \mathrm{GHz}$ Inverse Synthetic Aperture Radar," IEEE Transactions on Terahertz Science and Technology, vol. 3, no. 5, pp. 594-605, 2013.

24. B. Zhang, Y. Pi and J. Li, "Terahertz Imaging Radar With Inverse Aperture Synthesis Techniques: System Structure, Signal Processing, and Experiment Results,” IEEE Sensors Journal, vol. 15, no. 1, pp. 290-299, 2015 .

25. Z. Bao, M. Xing and T. Wang, Technology of Radar Imaging, Bei Jing: Publishing House of Electronics Industry, 2005, p. 336.

26. X. Li, G. Liu and J. Ni, "Autofocusing of ISAR images based on entropy minimization," IEEE Transactions on Aerospace and Electronic Systems, vol. 35, no. 4, pp. 1240-1252, 1999.

27. T. Zeng, R. Wang and F. Li, "SAR Image Autofocus Utilizing Minimum-Entropy Criterion," IEEE Geoscience and Remote Sensing Letters, vol. 10, no. 6, pp. 1552-1556, 2013.

28. Z. Sun, C. Li, X. Gao and G. Fang, "Minimum-Entropy-Based Adaptive Focusing Algorithm for Image Reconstruction of Terahertz Single-Frequency Holography With Improved Depth of Focus," IEEE Transactions on Terahertz Science and Technology, vol. 53, no. 1, pp. 519-526, 2015.

29. W. G. Carrara, R. S. Goodman and R. M. Majewski, Spotlight Synthetic Aperture Radar Signal Processing Algorithms, Boston London: Artech House, 1995.

30. D. M. Sheen, D. L. McMakin and T. E. Hall, "Three-dimensional millimeter-wave imaging for concealed weapon detection," IEEE Transactions on Microwave Theory and Techniques, vol. 49, no. 9, pp. 1581-1592, 2001.

31. J. Zdanevičius, M. Bauer, S. Boppel, V. Palenskis, A. Lisauskas and V. Krozer, et al., "Camera for HighSpeed THz Imaging," Journal of Infrared, Millimeter, and Terahertz Waves, 2015 